\section{P3.420 TARGETED VACCINATION PROGRAMME SUCCESSFUL IN REDUCING ACUTE HEPATITIS B IN MEN HAVING SEX WITH MEN IN AMSTERDAM, THE NETHERLANDS}

doi:10.1136/sextrans-2013-051184.0871

${ }^{1} \mathrm{G}$ G van Rijckevorsel, ' J Whelan, ${ }^{2,3} \mathrm{M}$ Kretzschmar, ${ }^{1} \mathrm{E}$ Siedenburg, ${ }^{1,4} \mathrm{G}$ Sonder, ${ }^{1,5} \mathrm{R}$ Geskus, ${ }^{2,3}$ R Coutinho, ${ }^{1,4}$ A van den Hoek. 'Public Health Service of Amsterdam, Amsterdam, The Netherlands; ${ }^{2}$ Centre for Infectious Disease Control, National Institute for Public Health and the Environment, RIVM, Bilthoven, The Netherlands; ${ }^{3}$ Julius Centre for Health Sciences and Primary Care, University Medical Centre Utrecht, Utrecht, The Netherlands; ${ }^{4}$ Academic Medical Centre, Department of Internal Medicine, Division of Infectious Diseases, Tropical Medicine and AIDS, Amsterdam, The Netherlands; ${ }^{5}$ Academic Medical Centre, Department of Clinical Epidemiology, Biostatistics and Bioinformatics, Amsterdam, The Netherlands

Background In the Netherlands, transmission of hepatitis B virus occurs mainly within behavioural high-risk groups, such as in men who have sex with men. Therefore, a vaccination programme has targeted these high-risk groups. Previous evaluations of this programme up until 2006 (incidence trend analysis, mathematical modelling and molecular sequence models) could prove no impact. This study evaluates the impact of the vaccination programme targeting Amsterdam's large population of men who have sex with men from 1998 through 2011.

Methods We used Amsterdam data from the national database of the vaccination programme for high-risk groups (January 1, 1998 to December 31, 2011). Programme and vaccination coverage were estimated with population statistics. Incidence of acute hepatitis B was analysed with notification data from the Amsterdam Public Health Service (1992 to 2011). Mathematical modelling accounting for vaccination data and trends in sexual risk behaviour was used to explore the impact of the programme.

Results At the end of 2011, programme coverage was estimated at $41 \%$ and vaccination coverage $30 \%$ to $38 \%$. Most participants (67\%) were recruited from the outpatient department for sexually transmitted infections and outreach locations such as saunas and gay bars. Incidence of acute hepatitis B dropped sharply after 2005. The mathematical model in which those who engage most in high-risk sex are vaccinated, best explained the decline in incidence.

Conclusions Transmission of hepatitis B virus among Amsterdam's men who have sex with men has decreased, despite ongoing high-risk sexual behaviour. International concern also exists about the effectiveness of such programmes when the uptake or coverage remains low. This study proves that a targeted vaccination programme can be effective with vaccination coverage below $40 \%$, as long as MSM who engage most in high-risk sex, such as clients of STI clinics, are reached.

\section{P3.421 CONTRIBUTIONS OF INTENSIVE HIV PREVENTION PROGRAMME IN INCREASING HIV TESTING AMONG MEN WHO HAVE SEX WITH MEN IN INDIA: A DECOMPOSITION ANALYSIS}

doi:10.1136/sextrans-2013-051184.0872

S Ramesh, P Mehrotra, N Saggurti, B Mahapthra, R Mishra. Population Council, New Delhi, India

Background and Objective The prevalence of HIV among MSM continues to be disproportionately high in India. The objective of this study was to identify the factors associated with change in the prevalence of HIV testing overtime and to assess their relative contribution in increasing (or decreasing) the prevalence of HIV testing among men who have sex with men.
Methods Data are drawn from two rounds of cross-sectional biobehavioural surveys of 7403 (round I: 3895, round II: 3508) selfidentified MSM from three states of India, recruited through probability based sampling in 2005-06 and 2009-10 respectively. Logistic regression models were used to assess the relationship between socio-demographic characteristics, sexual behaviours, programme exposure and HIV testing. Significant factors were further parsed using decomposition analysis to examine the contribution of different components of that factor towards the change in HIV testing over time.

Results There was a significant increase in the proportion of MSM reporting HIV testing from round I to round II (13\% vs. $77 \%$ : $p<0.001)$. Age, literacy, self-identified sexual identity, type of partner and exposure to intervention were significantly associated with HIV testing. Literates; those in the age groups of 25 to 34; kothis (predominantly receptive); those who had both commercial and non-commercial partners and those who were exposed to the intervention contributed the most to the increase in HIV testing over time.

Conclusions These study results underscore the potential of identifying and targeting segments within the at-risk MSM population which are currently not accessing HIV testing services. Voluntary HIV testing affects sexual behaviour leading to a positive motivation for safer sexual practises.

\section{P3.422 OPPORTUNITIES FOR HIV PREVENTION AMONG MEN WHO HAVE SEX WITH MEN IN THE UK: HIV TESTING AND WILLINGNESS TO USE PRE-EXPOSURE PROPHYLAXIS}

doi:10.1136/sextrans-2013-051184.0873

'L M McDaid, ${ }^{2} \mathrm{~A}$ Aghaizu, ${ }^{1} \mathrm{Y}$ Young, ${ }^{3} \mathrm{D}$ Mercey, ${ }^{3} \mathrm{~A}$ Copas, ${ }^{3} \mathrm{~A}$ M Johnson, ${ }^{3} \mathrm{G}$ Hart, ${ }^{2} \mathrm{~A}$ Nardone. 'Medical Research Council, Glasgow, UK; ${ }^{2}$ Health Protection Agency, London, UK: ${ }^{3}$ University College London, London, UK

Background Men who have sex with men (MSM) remain most at risk of acquiring HIV in the UK. While biomedical prevention methods, such as Pre-Exposure Prophylaxis (PrEP), present new prevention opportunities, questions remain over their acceptability and fit with existing prevention strategies and HIV risk management, including regular HIV testing. Here, we investigate the HIV testing behaviours and willingness to use PrEP among MSM in the UK.

Methods Cross-sectional surveys of HIV-negative MSM in gay social venues in Edinburgh, Glasgow and London in 2011 ( $N=2222)$. Results $53.2 \%(1177 / 2214)$ of participants had had an HIV test in the previous 12 months and 29.6\% (642/2167) reported $4+$ named HIV tests in the last two years. 48.2\% (1070/2221) reported that their most recent HIV test was a regular test or sexual health check. Among men at higher risk of HIV infection (reporting unprotected anal intercourse [UAI] with multiple, casual or unknown/discordant partners), 40.2\% (343/854) reported regular testing. Half of all participants would be willing to take PrEP on a daily basis $(52.3 \%, 1133 / 2166)$. In all three cities, willingness to take PrEP was associated with younger age and higher levels of sexual risk behaviour (UAI with multiple, casual or unknown/discordant partners in Edinburgh and Glasgow and UAI with casual partners in London), but with testing for HIV or other sexually transmitted infections (STIs) in the previous 12 months in Edinburgh and Glasgow only.

Conclusion PrEP could provide a new method of biomedical HIV prevention for men at high risk of acquiring infection, and there is a willingness to engage with this among MSM in the UK. However, this would require a level of frequent HIV testing beyond that currently reported by men at high risk. The potentially complex relationship between sexual risk behaviour, HIV testing, and PrEP use requires further research. 